Original

\title{
Adherencia al tratamiento de hipertensión arterial, hipercolesterolemia y diabetes en población anciana de una cohorte española
}

\author{
Aida Moreno Juste ${ }^{a, b, *}$, Antonio Gimeno Miguel ${ }^{a}$, Beatriz Poblador Plou ${ }^{a}$, \\ Francisca González Rubio ${ }^{\mathrm{a}, \mathrm{b}, \mathrm{c}}$, María Mercedes Aza Pascual-Salcedo ${ }^{\mathrm{a}, \mathrm{b}}$, Enrica Menditto ${ }^{\mathrm{d}}$ \\ y Alexandra Prados Torres ${ }^{\text {a }}$ \\ a Grupo EpiChron de Investigación en Enfermedades Crónicas, Instituto Aragonés de Ciencias de la Salud (IACS), IIS Aragón, REDISSEC ISCIII, Zaragoza, España \\ b Servicio Aragonés de Salud (SALUD), Zaragoza, España \\ ' Grupo de Trabajo de Utilización de Fármacos de la semFYC, España \\ d CIRFF, Centro de Farmacoeconomía, Universidad de Nápoles Federico II, Nápoles, Italia
}

\section{INFORMACIÓN DEL ARTÍCULO}

\section{Historia del artículo:}

Recibido el 10 de julio de 2018

Aceptado el 18 de octubre de 2018

On-line el xxx

\section{Palabras clave:}

Adherencia

Medicación

Polifarmacia

Multimorbilidad

Enfermedades crónicas

\section{R E S U M E N}

Antecedentes y objetivo: Varios estudios han puesto de manifiesto un cumplimiento terapéutico subóptimo en la población general, sobre todo en ancianos y en enfermos crónicos. El objetivo de este estudio es describir la adherencia al tratamiento de diabetes mellitus, dislipidemia e hipertensión arterial, e identificar los factores que la influencian.

Material y métodos: Estudio observacional transversal retrospectivo sobre 16.208 pacientes mayores de 65 años de la Cohorte EpiChron, que iniciaron tratamiento en monoterapia de un antidiabético, un hipolipidemiante o un antihipertensivo en 2010. La adherencia se midió mediante el cálculo de la relación de posesión de medicación durante un año de seguimiento, considerándose adherentes los casos con posesión de medicación $\geq 80 \%$. Se realizó un estudio descriptivo y un modelo de regresión logística para identificar los factores predictores de baja adherencia.

Resultados: La adherencia a los antidiabéticos, antihipertensivos e hipolipidemiantes fue del 72,4; 50,7 y $44,3 \%$, respectivamente. Se observó un aumento en la adherencia del 3-8\% por cada enfermedad crónica adicional del paciente. La presencia de enfermedad mental no afectó a la adherencia, y el sexo, edad y número de fármacos prescritos no presentaron efectos consistentes.

Conclusiones: Los resultados obtenidos ponen de manifiesto una adherencia al tratamiento subóptima en las enfermedades crónicas estudiadas. La adherencia aumentó con el número de enfermedades crónicas, mientras que sexo, edad y número de fármacos no presentaron un efecto consistente. Es necesario investigar si existen otros factores que puedan influir en la adherencia terapéutica, ya que su mejora puede tener mayor impacto en la salud que cualquier avance en las terapias.

(c) 2018 Elsevier España, S.L.U. Todos los derechos reservados.

\section{Adherence to treatment of hypertension, hypercholesterolaemia and diabetes in an elderly population of a Spanish cohort}

\section{A B S T R A C T}

Background and objective: Sub-optimal adherence to treatment in the general population has been highlighted in several studies, especially in the elderly and/or chronic patients. This study aims to describe the adherence to treatment of diabetes mellitus, dyslipidaemia and hypertension, and to identify the factors that influence adherence.

Material and method: Retrospective, cross-sectional observational study on 16,208 patients aged $\geq 65$ years from the EpiChron Cohort who initiated monotherapy treatment of an antidiabetic, a lipidlowering or an antihypertensive medication in 2010. Adherence was measured by calculating the

\footnotetext{
* Autor para correspondencia.

Correo electrónico: aidamorenoj@gmail.com (A. Moreno Juste).
} 
medication possession ratio during one year, considering those cases with medication possession ratio $\geq 80 \%$ to be adherent. We performed a descriptive study, and a logistic regression model was used to identify the predictors of low adherence.

Results: Adherence to antidiabetics, antihypertensive and lipid-lowering drugs was $72.4 \%, 50.7 \%$ and $44.3 \%$, respectively. An increase in adherence of 3-8\% was observed for each additional chronic disease suffered by the patient. The presence of mental illness did not affect adherence, and sex, age and number of prescribed drugs did not present consistent effects.

Conclusion: The results obtained show a sub-optimal adherence to treatment for the 3 chronic diseases studied. Adherence increased with the number of chronic diseases, while sex, age and number of drugs did not show a consistent effect. It is necessary to investigate if there are other factors that may influence therapeutic adherence, since improving adherence may have a greater impact on health than any progress in therapies.

(C) 2018 Elsevier España, S.L.U. All rights reserved.

\section{Introducción}

La Organización Mundial de la Salud (OMS) define la multimorbilidad como la presencia de 2 o más enfermedades crónicas en una misma persona ${ }^{1}$. Este fenómeno es muy incidente en la mayoría de los países y su repercusión es mayor en la población de más edad, en la que los problemas de salud se acumulan debido a la mejoría de las condiciones socioeconómicas y a los avances en el campo de la salud, que han permitido que una proporción significativa de la población sobreviva a enfermedades previamente mortales $^{2}$. En España, según la Encuesta Nacional de Salud, la denominada población anciana joven de 65-74 años padece un promedio de 2,8 problemas crónicos de salud, mientras que los mayores de 75 años tienen de media 3,2 enfermedades ${ }^{3}$. Por otra parte, la multimorbilidad se asocia con la polifarmacia o prescripción simultánea y prolongada de múltiples medicamentos, lo que implica una serie de consecuencias indeseables, como aumento del riesgo de utilización inadecuada de fármacos, aparición de sintomatología secundaria al incumplimiento, infrautilización de fármacos eficaces, interacciones farmacológicas y reacciones adversas ${ }^{3,4}$. El cumplimiento terapéutico, en términos de tomar medicamentos, seguir dietas o realizar cambios en el estilo de vida por parte del paciente, también puede verse afectado por la toma simultánea de varios fármacos ${ }^{5}$. Una de las formas de determinar el grado en que los pacientes siguen las instrucciones que acompañan a los tratamientos prescritos es medir la adherencia a la medicación ${ }^{6}$. Las enfermedades crónicas son las más afectadas por una baja adherencia: se estima que alrededor de un $50 \%$ de la población que presenta enfermedad crónica no toma su medicación de la forma prescrita. El estudio de los factores relacionados con una baja adherencia terapéutica es crucial para diseñar estrategias de prevención que sirvan para aumentar la efectividad del tratamiento, disminuir la morbimortalidad y mejorar la calidad de vida del paciente ${ }^{7}$.

El objetivo de este estudio fue determinar la adherencia terapéutica a los fármacos más utilizados para el tratamiento de diabetes mellitus tipo 2, hipercolesterolemia e hipertensión arterial en nuevos usuarios de estos medicamentos de 65 años o más, y estudiar su efecto sobre sexo, edad, número de fármacos y presencia de otras dolencias crónicas y enfermedad mental.

\section{Material y métodos}

\section{Diseño y población del estudio}

Estudio observacional transversal retrospectivo realizado en la Cohorte EpiChron que integra, de forma anonimizada, información demográfica, clínica, de dispensación de fármacos, uso de servicios y resultados en salud procedente de las historias clínicas y de bases de datos clínico-administrativas de los usuarios del Sistema Aragonés de Salud ${ }^{8}$. Este estudio cuenta con el dictamen favorable del Comité de Ética de la Investigación de la Comunidad de Aragón (PI16/088)

Los fármacos dispensados fueron codificados según el Sistema de Clasificación Anatómica Terapéutica Química (ATC) ${ }^{9}$. Se seleccionaron aquellos pacientes de la cohorte de 65 o más años de edad que iniciaron una prescripción en monoterapia oral entre el 1 de julio y el 31 de diciembre de 2010 de alguno de los siguientes: a) un fármaco antidiabético oral (biguanidas, A10BA, sulfonilureas, A10BB, inhibidores de la alfa-glucosidasa, A10BF, tiazolidinodionas, $\mathrm{A} 10 \mathrm{BG}$, inhibidores de la dipeptil peptidasa 4 , $\mathrm{A} 10 \mathrm{BH}, \mathrm{u}$ otros antidiabéticos orales, $\mathrm{A0BX}$ ); b) un fármaco hipolipidemiante (inhibidores de la HMG-CoA reductasa, C10AA, fibratos, $\mathrm{C} 10 \mathrm{AB}$, secuestrantes de ácidos biliares, C10AC, u otros modificadores de lípidos, (10AX) o c) un fármaco antihipertensivo del sistema renina-angiotensina (inhibidores de la enzima convertasa angiotensina, C09AA, o antagonistas de la angiotensina II, C09CA). Se excluyó a aquellos pacientes: a) con algún fármaco del mismo grupo a estudio prescrito en los 6 meses anteriores a su inclusión en el estudio; b) que no mantuvieron la monoterapia, ni en combinación farmacológica ni por separado; c) sin un año de datos validados de la prescripción del fármaco o d) con una única dispensación del fármaco a estudio.

Con estos criterios se obtuvo una muestra final de 16.208 pacientes, que fueron clasificados en cada uno de los grupos farmacológicos a estudio con base en el nuevo fármaco prescrito en la fecha índice, y seguidos durante un periodo de 12 meses.

\section{Variables}

Se registró el sexo y la edad de cada paciente, considerando la edad a fecha de su inclusión en el estudio, que se estratificó en 3 grupos (65-74, 75-84 y $\geq 85$ años). Se recogieron otras variables explicativas de los pacientes en los 6 meses anteriores a la inclusión en el estudio, como número de fármacos coprescritos, número de enfermedades crónicas y presencia de enfermedad mental. Como número de fármacos coprescriptos se incluyeron todos los códigos ATC, salvo ATC J (antiinfecciosos de uso sistémico) y ATC V (varios), ya que solo se consideraron los fármacos empleados de forma crónica. Los diagnósticos crónicos fueron codificados según la Clasificación Internacional de Atención Primaria y la Clasificación Internacional de Enfermedades y, posteriormente, se unificaron en Expanded Diagnostic Clusters (EDC) usando el software Johns Hopkins ACG $^{\circledR}$ System (versión 11.0, The Johns Hopkins University, Baltimore, MD, EE. UU.). Se consideró presencia de enfermedad mental a la presencia de alguno de los siguientes códigos diagnósticos: PSY01 (ansiedad, neurosis), PYS02 (abuso de sustancias), PSY04 (problemas del comportamiento), PSY05 (trastorno de 
déficit de atención), PSY07 (esquizofrenia y psicosis afectiva), PSY08(trastornos de personalidad), PSY09 (depresión), o NUR11 (demencia y delirios).

Como variable resultado, se cuantificó la adherencia terapéutica mediante la medición de la tasa de utilización del medicamento (MPR, por las siglas en inglés de medication possession ratio) para cada uno de los grupos farmacológicos a estudio. El cálculo del MPR es un método estándar para la evaluación de la adherencia y se define como el número de unidades farmacológicas dispensadas dividido para el número de unidades esperadas para el periodo de observación ${ }^{10}$. Se calcula como la proporción del número de días con tratamiento suministrado durante el período intencionado de tratamiento, es decir, $100 \times \sum$ (días suministrados)/365. El número de días en los que se suministró tratamiento se estimó según el número de comprimidos dispensados durante el año de seguimiento, asumiendo en nuestro caso que la posología del tratamiento fue de un comprimido al día. Una vez calculado el MPR, se creó la variable dicotómica "Adherencia al tratamiento (sí/no)» considerando que un paciente fue adherente si MPR $\geq 80 \%{ }^{11}$.

\section{Análisis estadístico}

Se realizó un estudio descriptivo de la población y de la adherencia terapéutica para cada grupo farmacológico a estudio. Una vez medida la adherencia de los nuevos usuarios para cada grupo de fármacos, se realizó un modelo de regresión logística binaria para valorar la relación entre adherencia (variable dependiente) y las variables independientes: sexo, edad (estratificada en los 3 grupos de edad), número de fármacos coprescritos, número de comorbilidades crónicas y presencia de enfermedad mental. Para cada media se calcularon los intervalos de confianza al 95\%, y en la regresión logística se consideró un nivel de significación estadística del 0,05. El conjunto de cálculos fue realizado en el software Stata (versión 12.0, StataCorp LLC, College Sation, TX, EE. UU.).

\section{Resultados}

La población a estudio estuvo conformada por 2.950 nuevos usuarios de antidiabéticos, 7.076 nuevos consumidores de hipolipidemiantes y 6.182 nuevos usuarios de antihipertensivos. La mayor parte de los nuevos usuarios de estos fármacos fueron pacientes de edades comprendidas entre 65 y 74 años en el caso de los hipolipidemiantes, y de entre 75 y 84 años en el caso de los antidiabéticos y antihipertensivos. En todos los grupos farmacológicos hubo una mayor proporción de mujeres que de hombres. El cálculo del MPR mostró adherencias al tratamiento que oscilaron entre un 44 y un $72 \%$ de los pacientes, en el caso de los nuevos usuarios de hipolipidemiantes y antidiabéticos, respectivamente (tabla 1).

\section{Tabla 1}

Incidencia de nuevos usuarios en monoterapia oral de antidiabéticos, hipolipidemiantes y antihipertensivos en pacientes de 65 años o más de la Cohorte EpiChron entre el 1 de julio y el 31 de diciembre de 2010, según edad y sexo, y adherencia terapéutica a cada uno de los grupos farmacológicos

\begin{tabular}{|c|c|c|c|}
\hline & Antidiabéticos & Hipolipidemiantes & Antihipertensivos \\
\hline \multicolumn{4}{|l|}{$\operatorname{Sexo}(n, \%)$} \\
\hline Mujer & $1.555(52,7)$ & $4.220(59,6)$ & $3.449(55,8)$ \\
\hline Hombre & $1.395(47,3)$ & $2.856(40,4)$ & $2.733(44,2)$ \\
\hline \multicolumn{4}{|l|}{$\operatorname{Edad}(n, \%)$} \\
\hline $65-74$ & $1.213(41,1)$ & $3.497(49,4)$ & $2.422(39,2)$ \\
\hline $75-84$ & $1.301(44,1)$ & $2.826(39,9)$ & $2.731(44,2)$ \\
\hline$\geq 85$ & $436(14,8)$ & $753(10,7)$ & $1.029(16,6)$ \\
\hline \multicolumn{4}{|c|}{ Adherencia al tratamiento ${ }^{\mathrm{a}}(\%)$} \\
\hline Sí & 72,4 & 44,3 & 50,7 \\
\hline
\end{tabular}

a Medida como tasa de utilización de la medicación (MPR). Un paciente se consideró adherente si MPR $\geq 80 \%$.
Tabla 2

Efecto del sexo, la edad, el número de fármacos prescritos, el número de enfermedades crónicas y la presencia de enfermedad mental sobre la adherencia al tratamiento en nuevos usuarios en monoterapia oral de antidiabéticos, hipolipidemiantes o antihipertensivos

\begin{tabular}{|c|c|c|}
\hline & OR (IC 95\%) $)^{\mathrm{a}}$ & $p$ \\
\hline \multicolumn{3}{|l|}{ Antidiabéticos } \\
\hline Sexo (mujer vs. hombre) & $1,01(0,862-1,19)$ & 0,883 \\
\hline \multicolumn{3}{|l|}{ Edad } \\
\hline $75-84$ vs. $65-74$ & $0,960(0,812-1,13)$ & 0,630 \\
\hline$\geq 85$ vs. $65-74$ & $0,832(0,655-1,06)$ & 0,133 \\
\hline N. fármacos de coprescritos & $0,959(0,942-0,977)$ & $<0,001$ \\
\hline$N .^{\circ}$ de enfermedades crónicas & $1,079(1,05-1,11)$ & $<0,001$ \\
\hline Presencia de enfermedad mental & $0,864(0,712-1,05)$ & 0,137 \\
\hline \multicolumn{3}{|l|}{ Hipolipidemiantes } \\
\hline Sexo (mujer vs. hombre) & $0,812(0,731-0,902)$ & $<0,001$ \\
\hline \multicolumn{3}{|l|}{ Edad } \\
\hline $75-84$ vs. $65-74$ & $0,858(0,770-0,957)$ & 0,006 \\
\hline$\geq 85$ vs. $65-74$ & $0,675(0,563-0,809)$ & $<0,001$ \\
\hline$N .^{\circ}$ de fármacos coprescritos & $1,02(1,01-1,03)$ & 0,002 \\
\hline $\mathrm{N} .^{\circ}$ de enfermedades crónicas & $1,07(1,05-1,09)$ & $<0,001$ \\
\hline Presencia de enfermedad mental & $0,924(0,813-1,05)$ & 0,221 \\
\hline \multicolumn{3}{|l|}{ Antihipertensivos } \\
\hline Sexo (mujer vs. hombre) & $0,877(0,785-0,980)$ & 0,021 \\
\hline \multicolumn{3}{|l|}{ Edad } \\
\hline $75-84$ vs. $65-74$ & $1,01(0,897-1,13)$ & 0,878 \\
\hline$\geq 85$ vs. $65-74$ & $0,949(0,808-1,12)$ & 0,529 \\
\hline$N .^{\circ}$ de fármacos coprescritos & $1,01(0,999-1,03)$ & 0,072 \\
\hline $\mathrm{N} .^{\circ}$ de enfermedades crónicas & $1,03(1,01-1,05)$ & 0,001 \\
\hline Presencia de enfermedad mental & $1,01(0,881-1,16)$ & 0,893 \\
\hline
\end{tabular}

a Para cada variable explicativa se proporciona el odds ratio (OR) de ser adherente, acompañado de su intervalo de confianza al $95 \%$.

El modelo de regresión logística aplicado en el grupo de nuevos usuarios de antidiabéticos mostró que la adherencia al tratamiento disminuye en un $4,1 \%$ por cada fármaco coprescrito a los antidiabéticos, mientras que por cada comorbilidad crónica concomitante aumenta la probabilidad de ser adherente en un 7,9\% (tabla 2). Por el contrario, el sexo, la edad y la presencia de enfermedad mental no modificaron la adherencia a este grupo farmacológico. En el grupo de nuevos consumidores de hipolipidemiantes, se observó que ser mujer disminuye la probabilidad de ser adherente en un $18,8 \%$ en comparación con ser hombre. Los pacientes entre 75 y 84 años presentaron una probabilidad de ser adherentes un $14,2 \%$ menor que el grupo de referencia de 65 a 74 años, mientras que en el caso de los mayores de 85 años esta probabilidad fue un 32,5\% menor. Cada comorbilidad crónica adicional presentada por los pacientes con hipolipidemiantes aumentó la probabilidad de ser adherente un $6,7 \%$, y con cada fármaco coprescrito se vio aumentada en un $2,2 \%$. Por último, los modelos de regresión logística revelaron una probabilidad un $12,3 \%$ menor de ser adherentes a los antihipertensivos en el caso de las mujeres. La probabilidad de ser adherentes aumentó en un 3,5\% con cada comorbilidad crónica añadida, mientras que no se observó relación con la edad, número de fármacos coprescritos ni presencia de enfermedad mental.

\section{Discusión}

Este trabajo estudia la adherencia terapéutica al tratamiento de 3 de las enfermedades crónicas más prevalentes en población aragonesa de 65 años o más, como son la hipertensión arterial, la hipercolesterolemia y la diabetes mellitus de tipo 2, así como los factores que la condicionan.

A pesar de la importancia de un buen cumplimiento terapéutico en el tratamiento de estas enfermedades crónicas, en nuestro estudio se observa en general una baja adherencia al tratamiento, sobre todo en el caso de los hipolipidemiantes y los antihipertensivos, en los que aproximadamente solo uno de cada 2 pacientes es adherente. Este hecho puede traducirse en un aumento de las 
complicaciones de la enfermedad a largo plazo y en un aumento de la morbimortalidad ${ }^{12}$. Otros estudios han arrojado niveles mayores de adherencia al tratamiento hipolipidemiante, como uno realizado en pacientes con enfermedad coronaria, donde la adherencia fue de un $79,8 \%$ de los casos a los 400 días de seguimiento ${ }^{13}$. En otro estudio realizado en el año 2010 , se reportó una adherencia al tratamiento con antihipertensivos del $79,8 \%$, pero esta fue medida con la técnica de la dosis diaria definida ${ }^{14}$. Esto, junto con posibles diferencias en los criterios de inclusión de los pacientes, podría justificar en parte las diferencias observadas con nuestro estudio.

Por el contrario, las cifras de adherencia a antidiabéticos revelaron que casi 3 de cada 4 pacientes son adherentes a su tratamiento. En un estudio de 2011 se encontraron resultados similares, con una adherencia a los antidiabéticos también superior a la observada con los hipolipidemiantes $(68,6 \text { vs. } 57,5 \%)^{15}$.

Con relación a los factores que influyen en la adherencia, se observó que en el grupo de nuevos usuarios de antihipertensivos e hipolipidemiantes la adherencia fue mayor en los hombres que en las mujeres. Este hecho ha sido interpretado por algunos autores considerando que la menor adherencia en mujeres es debido al papel de cuidadora principal de la familia, de forma que tienen menos tiempo y energía para su propio cuidado personal ${ }^{16}$. En cuanto a la edad, únicamente se relacionó de forma significativa con la probabilidad de adherencia en el grupo de hipolipidemiantes, en el que a mayor edad se observó una disminución drástica de la adherencia. En este sentido, no hay consenso sobre el papel de la edad en la adherencia, ya que en algunos estudios se ha observado un aumento de la adherencia conforme aumenta la edad, mientras que otros indican lo contrario ${ }^{17}$. Por otra parte, no existen estudios que investiguen las causas de la falta de adherencia a los hipolipidemiantes en prevención primaria. Algunas hipótesis sobre este hecho que se han propuesto recientemente especulan sobre que la falta de adherencia a estos fármacos podría ser debida a los efectos adversos sufridos por los pacientes y no reconocidos por los profesionales como tales, de forma que los propios pacientes abandonan el tratamiento al no encontrarse bien con la toma de los fármacos.

Además del sexo y la edad, también se han relacionado con la adherencia el número de fármacos y las comorbilidades concomitantes, la presencia de sintomatología, el hecho de que se trate de un tratamiento curativo o preventivo, el conocimiento de la enfermedad y la presencia de enfermedad mental, entre otros. La falta de motivación y la disminución de la atención y la memoria han sido valorados también como causa de baja adherencia en algunos estudios $^{14}$, aunque en nuestro caso no se han estudiado estos factores, ya que no se dispone de información al respecto. En nuestro estudio, el efecto sobre la adherencia de otros fármacos administrados de forma simultánea no fue consistente. En la bibliografía se ha observado cómo la complejidad del tratamiento es inversamente proporcional a la adherencia ${ }^{14}$. En un estudio realizado sobre adherencia al tratamiento con antihipertensivos en Nueva Jersey se observó que tener prescritos más de 8 fármacos disminuía la probabilidad de adherencia ${ }^{18}$. De forma similar, en un estudio en la comunidad autónoma de Aragón en 2010 también se concluyó que la polifarmacia se asocia a una baja adherencia al tratamiento. Entre los factores que se han relacionado en este aspecto con una disminución de la adherencia destacan la prescripción de un gran número de fármacos, la dispensación de medicación de forma no sincronizada, la frecuencia de la administración, la variabilidad en las dosis y las instrucciones especiales a la hora de tomar la medicación ${ }^{14}$.

Al contrario del efecto del número de fármacos coprescritos, que no fue consistente, sí que se observa en este estudio un aumento de la adherencia terapéutica conforme aumenta el número de enfermedades crónicas que presenta el paciente, independientemente del grupo farmacológico estudiado. La adherencia al tratamiento aumentó en término medio entre un 3 y un $8 \%$ por cada comorbilidad crónica adicional. El efecto sobre la adherencia terapéutica de la presencia de múltiples enfermedades crónicas o multimorbilidad ha sido ampliamente estudiado, valorándose la presencia de asociaciones positivas entre enfermedades y la adherencia a la medicación antihipertensiva ${ }^{19}$. En un estudio sobre hipolipidemiantes, la adherencia aumentó del 56\% en los pacientes con una sola comorbilidad crónica al $72 \%$ en pacientes con 3 o más comorbilidades ${ }^{20}$. Por el contrario, algunos estudios han demostrado que la presencia de otras comorbilidades está asociada a una disminución de la adherencia ${ }^{21,22}$. En otro trabajo, se observó que las comorbilidades que requerían un consumo regular de medicamentos no afectaron el cumplimiento de los pacientes con antihipertensivos ${ }^{22}$. También se ha señalado que la sintomatología influye de forma positiva en el cumplimiento terapéutico ya que, al presentar sintomatología, los pacientes son más conscientes de la enfermedad y toman la medicación para evitarla, por lo que se adhieren más al tratamiento establecido. Así, es fácil de entender que existan altas tasas de incumplimiento en procesos asintomáticos como la dislipidemia ${ }^{23}$. Otra de las causas de buena adherencia puede ser que el consumo de este tipo de fármacos se deba a una prevención secundaria. En varios estudios se ha observado que en prevención primaria existe una mayor tasa de no cumplimiento que en la prevención secundaria, así como que la presencia de comorbilidades cardiovasculares o complicaciones derivadas de la enfermedad mejoran la adherencia ${ }^{24,25}$. Se ha observado una mayor adherencia al tratamiento de la hipertensión arterial en pacientes con mayor riesgo cardiovascular, probablemente debido a una mayor conciencia de la importancia del tratamiento por parte tanto del paciente como de su médico de Atención Primaria, así como a una mayor motivación para seguir el curso del tratamiento ${ }^{14}$.

La heterogeneidad en los resultados y conclusiones de los diferentes estudios puede estar relacionada con el hecho de que la adherencia a la medicación es un fenómeno complejo influido por varios factores, incluyendo el conocimiento sobre la enfermedad, la actitud de los pacientes, el entorno socioeconómico, los problemas del sistema de salud, la relación médico-paciente y el papel de los farmacéuticos ${ }^{22,26}$. Este hecho se ha corroborado en un estudio basado en entrevistas a pacientes con diabetes mellitus de tipo 2 , que señala que la mayoría de estos pacientes entienden la importancia de tomar medicamentos y al mismo tiempo reconocen la importancia de estar adecuadamente informados sobre su enfermedad, las comorbilidades y los beneficios de la medicación ${ }^{27}$.

\section{Fortalezas y limitaciones}

La principal fortaleza de este estudio es que se trata de un estudio de base poblacional a gran escala realizado en una cohorte validada para investigación en enfermedades crónicas y fármacoepidemiología, en la que los datos son tratados mediante un control de calidad continuo. Además, la inclusión en el estudio únicamente de pacientes de nueva prescripción de cada uno de los fármacos estudiados minimiza el riesgo de aparición de sesgos de confusión.

Entre las principales limitaciones destaca que, para medir la adherencia al tratamiento, se considera que cada paciente consume un comprimido del fármaco al día. La mayor dosificación diaria en algunos casos produciría una sobrestimación de la adherencia, ya que los pacientes con más de una dispensación pueden dejar de tomar la medicación o consumirla de forma incorrecta y ser considerados adherentes. También puede ocurrir lo contrario y que la dosis diaria sea menor a un comprimido, que haga que la receta dispensada dure más días de lo calculado y se produzca una infraestimación de la adherencia. Además, se supone que la dispensación para la misma enfermedad es igual para todos los pacientes, lo que no se corresponde con la práctica clínica en la que la prescripción se realiza de forma individualizada según la tolerabilidad de cada paciente, los comportamientos de la enfermedad o las interacciones 
con otros fármacos ${ }^{28}$. El método del cálculo del MPR para valorar la adherencia asume que los comprimidos dispensados son consumidos por el paciente, aunque este método se acepta como estándar para medir la adherencia debido a la dificultad que supone medir el consumo real de la medicación ${ }^{29}$. Por otra parte, se desconocen tanto las causas de la interrupción del tratamiento, que podría deberse a la aparición de efectos adversos o a la percepción de una baja eficacia ${ }^{20,30}$, como las indicaciones del tratamiento para valorar de forma más exhaustiva la adherencia terapéutica. Asimismo, este estudio no incluye información sobre algunas otras variables que también podrían influir la adherencia al tratamiento, como son la función física, la fragilidad o la discapacidad.

\section{Conclusiones}

Los resultados obtenidos ponen de manifiesto una adherencia subóptima al tratamiento en las 3 enfermedades crónicas estudiadas. En cuanto a los factores que modulan la adherencia, se ha observado que la adherencia aumenta con el número de enfermedades crónicas, mientras que el sexo, la edad, la enfermedad mental y el número de fármacos no presentan un efecto consistente sobre la adherencia. Este estudio es exploratorio e invita a la realización de otros para valorar la adherencia terapéutica en el caso de combinaciones de fármacos y, de forma más exhaustiva, el papel que ejercen factores como los efectos adversos, el conocimiento del paciente sobre la importancia del correcto tratamiento de las enfermedades crónicas, el entorno social del paciente, o si se trata de prevención primaria o secundaria. Los resultados obtenidos podrían ayudar a la puesta en marcha de actuaciones sobre el paciente dirigidas a mejorar la adherencia terapéutica a las enfermedades crónicas y a evitar así las consecuencias negativas del incumplimiento terapéutico ya que, como afirma la OMS, mejorar la adherencia terapéutica puede tener un mayor impacto en la salud que cualquier avance en las terapias.

\section{Fuentes de financiación}

La presente investigación no ha recibido ayudas específicas provenientes de agencias del sector público, sector comercial ni entidades sin ánimo de lucro.

\section{Conflictos de intereses}

Ninguno.

\section{Agradecimientos}

Los autores agradecen su colaboración a los miembros de Action Group A1 del European Innovation Partnership on Active and Healthy Ageing (EIP-AHA).

\section{Bibliografía}

1. Gauld R, Blank R, Burgers J, Cohen AB, Dobrow MK, Ikegami NK, et al. The World Health Report 2008 - Primary healthcare: How wide is the gap between its agenda and implementation in 12 high-income health systems? Health Policy. 2012;7:38-58.

2. Prados-Torres A, Poblador-Plou B, Calderón-Larrañaga A, Gimeno-Feliu LA, González-Rubio F, Poncel-Falcó A, et al. Multimorbidity patterns in primary care: Interactions among chronic diseases using factor analysis. PLoS One. 2012;7, e32190.

3. Prados-Torres A, del Cura-González I, Prados-Torres D, López-Rodríguez JA, Leiva-Fernández F, Calderón-Larrañaga A, et al. Effectiveness of an intervention for improving drug prescription in primary care patients with multimorbidity and polypharmacy: Study protocol of a cluster randomized clinical trial (MultiPAP project). Implement Sci. 2017;12:54

4. Calderón-Larrañaga A, Gimeno-Feliu LA, González-Rubio F, Poblador-Plou B, Lairla-San José M, Abad-Díez JM, et al. Polypharmacy patterns: Unravelling systematic associations between prescribed medications. PLoS One. 2013;8, e84967

5. Vermiere E, Hearnshaw H, Van Royen PD. Patient adherence to treatment: Three decades of research: A comprehensive review. J Clin Pharm Ther. $2001 ; 26: 331-42$.

6. Calderón-Larrañaga A, Poblador-Plou B, González-Rubio F, Gimeno-Feliu LA, Abad-Díez JM, Prados-Torres A. Multimorbidity, polypharmacy, referrals, and adverse drug events: Are we doing things well? Br J Gen Pract. 2012;62:e821-6.

7. World Health Organization. Adherence to long-term therapies: Evidence for action [Internet]. 2015 [consultado 3 Jul 2018]. Disponible en: http://www.who.int/chp/knowledge/publications/adherence_report/en/.

8. Prados-Torres A, Poblador-Plou B, Gimeno-Miguel A, Calderón-Larrañaga A, Poncel-Falcó A, Gimeno-Feliú LA, et al. Cohort Profile: The Epidemiology of Chronic Diseases and Multimorbidity. The EpiChron Cohort Study. Int J Epidemiol. 2018;0(0.).

9. World Health Organization. Collaborating Centre for Drugs Statistics Methodology - ATC/DDD Index [Internet]. [consultado 19 Mar 2018]. Disponible en: https://www.whocc.no/atc_ddd_index/.

10. Menditto E, Orlando V, Malo-Fumanal S, Prados-Torres A, Cahir C. Measuring medication adherence in health-related databases. En: Costa E, editor. Adherence to Medical Plans for Active and Healthy Ageing. Nueva York: Nova Science Publisher; 2017. p. 189-206

11. O'Shea MP, Teeling M, Bennet. An observational study examining the effect of comorbidity on the rates of persistence and adherence to newly initiated oral anti-hyperglycaemic agents. Pharmacoepidemiol Drug Saf. 2013;22, 1336-1244.

12. Alefishat EA, Abu Farha RK, Al-Debei MM. Self-reported adherence among individuals at high risk of metabolic syndrome: Effect of knowledge and attitude. Med Princ Pract. 2017;26:157-63.

13. Chi MD, Vansomphone SS, Liu ILA, Cheetham TC, Green KR, Scott RD, et al. Adherence to statins and LDL-cholesterol goal attainment. Am J Manag Care. 2014;20:e105-12.

14. Calderón-Larrañaga A, Diaz E, Poblador-Plou B, Gimeno-Feliu LA, Abad-Díez JM, Prados-Torres A. Non-adherence to antihypertensive medication: The role of mental and physical comorbidity. Int J Cardiol. 2016;207:310-6.

15. Zhang Q, Zhao C, Davies MJ, Radican L, Seck T. Compliance and persistence with concomitant statin and oral antihyperglycemic therapy. Am J Manag Care. 2011;17:746-52.

16. Rolnick SJ, Pawloski PA, Hedblom BD, Asche SE, Bruzek RJ. Patient characteristics associated with medication adherence. Clin Med Res. 2013:11:54-65.

17. Pedan A, Varasteh L, Schneeweiss S. Analysis of factors associated with statin adherence in a hierarchical model considering physician, pharmacy, patient, and prescription characteristics. J Manag Care Pharm. 2007;13:487-96.

18. Monane M, Bohn RL, Gurwitz JH, Glynn RJ, Levin R, Avorn J. The effects of initial drug choice and comorbidity on antihypertensive therapy compliance. Results from a population-based study in the elderly. Am J Hypertens. 1997;10: 697-704.

19. Lee C-Y, Huang C-C, Shih H-C, Huang K-H. Factors influencing antihypertensive medication compliance in Taiwan: A nationwide population-based study. Eur J Prev Cardiol. 2013;20:930-7.

20. Latry P, Molimard M, Dedieu B, Couffinhal T, Bégaud B, Martin-Latry K. Adherence with statins in a real-life setting is better when associated cardiovascular risk factors increase: A cohort study. BMC Cardiovasc Disord. 2011;11:46.

21. McGovern A, Hinton W, Calderara S, Munro N, Whyte M, de Lusignan S. A class comparison of medication persistence in people with type 2 diabetes: A retrospective observational study. Diabetes Ther. 2018;9:229-42.

22. Saadat Z, Nikdoust F, Aerab-Sheibani H, Bahremand M, Shobeiri E, Saadat H, et al. Adherence to antihypertensives in patients with comorbid condition. Nephrourol Mon. 2015;7, e299863.

23. Orueta R, Toledano P, Gómez-Calcerrada RM. Actualización en Medicina de Familia: Cumplimiento terapéutico. Med Fam Semer. 2008:34:235-43.

24. Saarti S, Hajj A, Karam L, Jabbour H, Sarkis A, El Osta N, et al. Association between adherence, treatment satisfaction and illness perception in hypertensive patients. J Hum Hypertens. 2016;30:341-5.

25. Sanfélix-Gimeno G, Peiró S, Ferreros I, Pérez-Vicente R, Librero J, Catalá-López $\mathrm{F}$, et al. Adherence to evidence-based therapies after acute coronary syndrome: A retrospective population-based cohort study linking hospital, outpatient, and pharmacy health information systems in Valencia, Spain. J Manag Care Pharm. 2013;19:247-57

26. Menditto E, Cahir C, AzaPascual-Salcedo M, Bruzzese D, Poblador-Plou B, Malo $\mathrm{S}$, et al. Adherence to chronic medication in older populations: Application of a common protocol among three European cohorts. Patient Prefer Adherence. 2018;12:1975-87.

27. Labrador Barba E, Rodriguez de Miguel M, Hernández Mijares A, Alonso-Moreno FJ, Orera Peña ML, Aceituno S, et al. Medication adherence and persistence in type 2 diabetes mellitus: Perspectives of patients, physicians and pharmacists on the Spanish health care system. Patient Prefer Adherence. 2017;11: 707-18.

28. American Diabetes Association. 8. Pharmacologic approaches to glycemic treatment: Standards of medical care in diabetes-2018. Diabetes Care. 2017;41(Supp 1):S64-S74.

29. Halpern MT, Khan ZM, Schmier JK, Burnier M, Caro JJ Cramer J, et al. Recommendations for evaluating compliance and persistence with hypertension therapy using retrospective data. Hypertension. 2006;47:1039-48.

30. Ah YM, Lee JY, Choi YJ, Kim B, Choi KH, Kong J, et al. Persistence with antihypertensive medications in uncomplicated treatment-naïve patients: Effects of initial therapeutic classes. J Korean Med Sci. 2015;30:1800-6. 